\title{
Infantile onset panniculitis with uveitis and systemic granulomatosis
}

INSERM

\section{Source}

INSERM. (1999). Orphanet: an online rare disease and orphan drug data base. Infantile onset panniculitis with uveitis and systemic granulomatosis. ORPHA:251304

Infantile onset panniculitis with uveitis and systemic granulomatosis is a rare granulomatous autoinflammatroy disease characterized by infantile-onset, widespread, chronic, recurrent, progressive, lobular panniculitis associated with panuveitis, arthritis and severe systemic granulomatous inflammation. 